\title{
SOME SIMPLE EXAMPLES OF SYMPLECTIC MANIFOLDS
}

\author{
W. P. THURSTON
}

ABSTRACT. This is a construction of closed symplectic manifolds with no Kaehler structure.

A symplectic manifold is a manifold of dimension $2 k$ with a closed 2 -form $\alpha$ such that $\alpha^{k}$ is nonsingular. If $M^{2 k}$ is a closed symplectic manifold, then the cohomology class of $\alpha$ is nontrivial, and all its powers through $k$ are nontrivial. $M$ also has an almost complex structure associated with $\alpha$, up to homotopy.

It has been asked whether every closed symplectic manifold has also a Kaehler structure (the converse is immediate). A Kaehler manifold has the property that its odd dimensional Betti numbers are even. H. Guggenheimer claimed [1], [2] that a symplectic manifold also has even odd Betti numbers. In the review [3] of [1], Liberman noted that the proof was incomplete. We produce elementary examples of symplectic manifolds which are not Kaehler by constructing counterexamples to Guggenheimer's assertion.

There is a representation $\rho$ of $Z \oplus Z$ in the group of diffeomorphisms of $T^{2}$ defined by

$$
(1,0) \stackrel{\rho}{\longrightarrow} \mathrm{id}, \quad(0,1) \stackrel{\rho}{\longrightarrow}\left[\begin{array}{ll}
1 & 1 \\
0 & 1
\end{array}\right]
$$

where "[ $\left[\begin{array}{ll}0 & 1 \\ 0 & 1\end{array}\right]$ " denotes the transformation of $T^{2}$ covered by the linear transformation of $\mathbf{R}^{2}$. This representation determines a bundle $M^{4}$ over $T^{2}$, with fiber $T^{2}: M^{4}=\tilde{T}^{2} \times_{Z \oplus Z} T^{2}$, where $Z \oplus Z$ acts on $\tilde{T}^{2}$ by covering transformations, and on $T^{2}$ by $\rho\left(M^{4}\right.$ can also be seen as $\mathbf{R}^{4}$ modulo a group of affine transformations). Let $\Omega_{1}$ be the standard volume form for $T^{2}$. Since $\rho$ preserves $\Omega_{1}$, this defines a closed 2-form $\Omega_{1}^{\prime}$ on $M^{4}$ which is nonsingular on each fiber. Let $\rho$ be projection to the base: then it can be checked that $\Omega_{1}^{\prime}+p^{*} \Omega_{1}$ is a symplectic form. (It is, in general, true that $\Omega_{1}^{\prime \prime}+K \rho^{*} \Omega_{1}$ is a symplectic form, for any closed $\Omega_{1}^{\prime \prime}$ which is a volume form for each fiber, and $K$ sufficiently large.) But $H_{1}\left(M^{4}\right)=Z \oplus Z \oplus Z$, so $M^{4}$ is not a Kaehler manifold.

Many more examples can be constructed. In the same vein, if $M^{2 k}$ is a closed symplectic manifold, and if $N^{2 k+2}$ fibers over $M^{2 k}$ with the fundamental class of the fiber not homologous to zero in $N$, then $N$ is also a symplectic manifold. If, for instance, the Euler characteristic of the fiber is not zero, this

Received by the editors July 31, 1974.

AMS (MOS) subject classifications (1970). Primary 57D15, 58H05.

C American Mathematical Society 1976 
hypothesis is satisfied. To do this, one must see that if there is a closed 2-form $\alpha_{1}$ whose integral on a fiber is nonzero, then $\alpha_{1}$ is cohomologous to a 2 -form $\alpha$ which is nonsingular on each fiber. To find $\alpha$, first find a 2 -form $\beta$, not necessarily closed, which is nonsingular on each fiber, and whose integral on each fiber agrees with that of $\alpha_{1}$ : this exists by convexity considerations. On each fiber, $F$, there is a form $\gamma_{F}$ such that $\beta_{F}-\left(\alpha_{1}\right)_{f}=d\left(\gamma_{F}\right)$. This equation can also be solved differentiably in a small neighborhood of the base, so, by convexity considerations, there is a global 1-form $\gamma$ such that on each fiber, $\beta_{F}-\left(\alpha_{1}\right)_{F}=d\left(\gamma_{F}\right)$. Let $\alpha=\alpha_{1}+d(\gamma)$. If $\Omega_{1}$ is a symplectic form for $M^{2 k}$, then $\Omega=\alpha+K\left(\rho^{*} \Omega_{1}\right)$ is a symplectic form for $N^{2 k+2}, K$ is sufficiently large.

This construction, although it applies only to a narrow range of examples, nonetheless has a certain amount of flexibility. This leads me to make the

CONJECTURE. Every closed $2 k$-manifold which has an almost complex structure $\tau$ and a real cohomology class $\alpha$ such that $\alpha^{k} \neq 0$ has a symplectic structure realizing $\tau$ and $\alpha$.

I would like to thank Alan Weinstein for pointing out this question and for helpful discussions.

\section{REFERENCES}

1. H. Guggenheimer, Sur les variétés qui possèdent une forme extérieure quadratique dermée, C. R. Acad. Sci. Paris 232 (1951), 470-472. MR 12, 535.

2. - Variétés symplectiques, Colloq. Topologie de Strasbourg, 1951 (mimeographed notes).

3. P. Liberman, review of [1], Zentralblatt für Mathematik 54 (1956), 68.

Department of Mathematics, Fine Hall, Princeton University, Princeton, New Jersey 08540

Current address: School of Mathematics, Institute for Advanced Study, Princeton, New Jersey 08540 